\title{
Luxation du coude compliquée d'une fracture-avulsion de l'épicondyle médial chez un enfant
}

\section{Elbow dislocation combined with avulsion of medial epicondyle in a child}

\section{Tourdias}

Reçu le 26 septembre 2014; accepté le 10 novembre 2014

(C) SFMU et Lavoisier SAS 2014

Un jeune gymnaste de 12 ans est admis aux urgences pour une vive douleur du coude droit. Lors d'exercices aux agrès, il a été victime d'une chute à l'origine d'un violent traumatisme sur la main, le coude étant en hyperextension et l'avant-bras en supination. La présentation clinique bruyante permet d'évoquer une luxation postérieure avec à l'inspection une déformation caractéristique (Fig. 1A) et à la palpation une perte des repères osseux normaux. Il n'existe pas de complication vasculonerveuse, ni cutanée. Le bilan radiographique retrouve une luxation huméro-antébrachiale complète à déplacement postérieur pur, associée à une fracture-avulsion du noyau apophysaire de l'épicondyle médial (Fig. 1B, C). La réduction est effectuée par manœuvres externes puis une ostéosynthèse du fragment épicondylien médial est réalisée afin de le stabiliser. La luxation du coude représente la première luxation d'une articulation majeure chez l'enfant, et la deuxième chez l'adulte, survenant fréquemment au cours d'accidents sportifs [1]. La fracture de l'épicondyle médial constitue la complication la plus fréquente lors d'une luxation du coude mais passe malheureusement souvent inaperçue [1]. Elle se retrouve essentiellement chez l'adolescent où elle prend volontiers la forme d'un arrachement apophysaire lié à la zone de faiblesse que représente le cartilage de croissance non encore fusionné à ce niveau. Il n'est pas rare que ce fragment épicondylien avulsé se retrouve incarcéré dans l'interligne huméroulnaire. Il est alors important de le différencier d'un noyau épiphysaire huméral car ce déplacement intra-articulaire oblige à une réduction chirurgicale de la luxation par arthrotomie. La découverte d'une fracture-avulsion de l'épicondyle médial nécessite, après la réduction de la luxation, de

D. Tourdias $(\bowtie)$

Service d'accueil des urgences, SMUR, UHCD,

centre hospitalier Sud Gironde, rue Paul Langevin,

F-33210 Langon

e-mail : tourdiasdamien@yahoo.fr

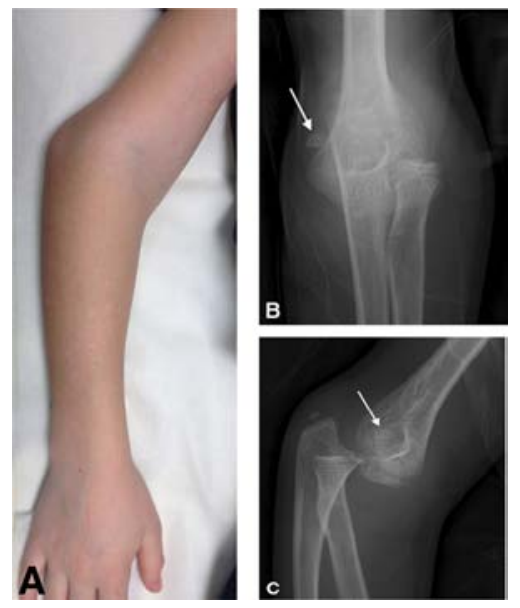

Fig. 1 Luxation complexe du coude. A. Aspect clinique classique d'une luxation huméro-antébrachiale postérieure : attitude en semiflexion et pronation avec la présence d'une saillie postérieure de l'olécrane associé à un élargissement antéropostérieur du coude. B. Cliché du coude de face. C. Cliché du coude de profil. Ces radiographies permettent d'objectiver la luxation postérieure et son caractère compliqué avec un noyau apophysaire épicondylien médial avulsé (flèches)

réaliser une ostéosynthèse du fragment avulsé. Cette fixation chirurgicale de l'épicondyle est en effet indispensable car il s'agit de la zone d'insertion du ligament collatéral ulnaire dont le rôle dans la stabilité de l'articulation du coude est essentiel.

\section{Référence}

1. Parsons BO, Ramsey ML (2010) Acute elbow dislocations in athletes. Clin Sports Med 29:599-609 